\section{SP0185 DON'T DELAY, CONNECT TODAY IN TWELVE VIDEO'S: HOW TO GET EARLY DIAGNOSIS OF RMD'S ON THE TABLE IN POLAND}

Jolanta Grygielska. Polish Rheuma Federation 'REF', National Board, Warsaw, Poland

Background: Early diagnosis and appropriate treatment is a mile stone in future progress of rheumatic and musculoskeletal disease (RMD), especially inflammatory. We know from our experiences that delays of diagnosis are connected with knowledge and attitude of patient, of primary care physician (fast referral to rheumatologist) and with access to rheumatologist. New EULAR campaign "Don't delay, connect today" gives an opportunity to pay attention to the problem of RMDs, increase public awareness and remind persons about responsibility for their own health.

Objectives: Aim of our project was to increase public awareness about RMDs and self-awareness of patients, to present how important is a role of everybody being face to face with progressing disease. It was most important to reach to younger people believing that rheumatic diseases are typical only for older people. Lack of knowledge about RMDs and faith in myths are a first step to loose a time from first symptoms of diseases to appropriate diagnosis. The first step was translation of EULAR campaign slogan into Polish and preparing of Polish version of logo. It was done very fast after launching EULAR campaign.

Methods: We have many ideas which subject are more important for people around diagnosis. We decided to start with choice of subjects and connecting them with the first letter of Polish names of months. We tried to order them in logical process: from self-awareness to the sin of neglect though subject like for example medicine, physician, medication, choice, love, return. After decision about subjects we invited speakers for every subject. There were rheumatologists from National Institute of Geriatrics, Rheumatology and Rehabilitation in Warsaw and from other rheumatological clinics in Poland, mainly co-operating with our member associations. Every invited speaker received only a subject and main message to present in his/her film. Form, place and comments were suggested by speaker and discussed with a team preparing videos. Part of films were supported with our regional member associations. Forms of videos prepared in regions were decided by associations. In two films members of associations present their opinions and experiences to give a strength for new rheumatic persons to live with RMD and avoid unnecessary health situations. One video was enriched by physical exercises presented by students of physiotherapy under supervise of rheumatologist. All videos were filmed and prepared by our member, translation was done by another our member - both are professionals on their fields and with experiences of living with RMD. It gives added value to our campaign. After that videos were posted on our channel on YouTube and information about them was shared by our websites, facebook and Twitter. We sent information by e-mail to our colleagues and supporters. We invite every viewer to subscribe our channel to have easy access to our newest films.

Results: "Video for every month" is a series but every video can be used separately. Order of videos watching can be optional according to the viewer's choice. At the same time when videos were posted one by one, project on early diagnosis of rheumatoid arthritis supported by European founds and with our partnership start in Poland. Thanks to this our message "Don't delay, connect today" is stronger. Parallel our new project "RA - don't resign" starts from World Arthritis Day 2018. All actions give an occasion to focus on early diagnosis and appropriate treatment of RMDs.

Conclusion: Every aim can be achieve in co-operation and engagement of main players. We involve in our project members of regional associations, rheumatologists, students. During realization of other projects we used every occasion to share information about our videos. All subjects of videos are overtime. Every patient with RMD can receive new knowledge about her/his disease from reliable source everywhere and at every time in two languages versions Polish (voice) and English (subtitles). We are planning to use this form of information in next project.

Disclosure of Interests: None declared

DOI: 10.1136/annrheumdis-2019-eular.8488

\section{SP0186 WHAT DO GP'S KNOW ABOUT RMD'S? \#CONNECTTODAY IN MALTA}

Mary Vella. Arthritis and Rheumatism Association Malta (ARAM), NGO, Mgarr, Malta

Background: The Arthritis and Rheumatism Association Malta known as ARAM has been established these last eleven years since 2007. Through this experience as a support group working very closely with RMDs patients through various talks and seminars, and participating on Live Radio and Television to bring awareness on RMDs, ARAM became aware that there is Lack of Knowledge on the subject by the GPs and this needed to be addressed. Patients were being given the idea that once one develops arthritis there was nothing that can be done to embrace an independent and good quality of life. Obviously, with so much research and studies, this is not so!

I can confirm this myself being a patient who developed Rheumatoid Arthritis sixteen years ago. It was then that ARAM was established and being the President of the Association I advocated strongly for RMD Patients throughout the island. I believed that education and Knowledge on Arthritis should be a basic step both for the Patients as well as for the GPS and the General Public.

Objectives: When Eular announced the award for national campaign Don't delay Connect today' for early signs and symptoms and early referral, ARAM applied for the award in collaboration with the College of Family Doctors to educate the GPS on arthritis. This was possible after a number of meetings and discussion.

Methods: ARAM approached the Synapse which is an E-platform led by a medical doctor for Health Professionals based on sharing academic research and other articles to people that matter. With such a professional body ARAM produced four videos by Rheumatologist specialist on RMDs focusing on different aspects of these chronic conditions.

1. General Rheumatology Introduction -Dr Michaela Frendo

2. Juvenile Arthritis - Dr Cecilia Mercieca

3. Scleroderma- Dr Bernard Coleiro

4. Fibromyalgia- Prof Andrew Borg

Another two educational Talks for GPs led by Prof Andrew Borg, Head of Rheumatology Department of Mater Dei Hospital were held in a specific venue and were well attended. Topics delivered focused on:

1. Management of Inflammatory Joints

2. Management of Metabolic Bone diseases

Conclusion: To this effect, ARAM believes that GPs are now better informed and educated and more aware of possible early interventions that could mitigate the seriousness on the condition.

The general outcome of these talks include:

1. GPs became more educated and informed which will translate into

2. Better outcomes for the patients.

Results: Evaluation was carried out following both interventions by asking GPs for their feedback on both the Synapse e-platform and the two seminars.

Conclusion: To this effect, ARAM believes that GPs are now better informed and educated and more aware of possible early interventions that could mitigate the seriousness on the condition.

Disclosure of Interests: None declared

DOI: 10.1136/annrheumdis-2019-eular.8614

SATURDAY, 15 JUNE 2019

09:00:00 - 10:30:00

Tackling inflammatory bone disorders in children and adults

\section{SP0187 \\ PATHOPHYSIOLOGY AND THERAPEUTIC CONSEQUENCES AUTO-INFLAMMATORY BONE DISORDERS}

Christian Hedrich. Institute of Translational Medicine, University of Liverpool,

Department of Women's and Children's Health, Liverpool, United Kingdom

Chronic nonbacterial osteomyelitis (CNO) is an autoinflammatory bone disorder, covering a clinical spectrum with asymptomatic inflammation of single bones at the one end, and chronic recurrent multifocal osteomyelitis (CRMO) at the other end. Bone inflammation, however, can also be a symptom of other autoimmune/ inflammatory conditions. Rare monogenic autoinflammatory diseases with bone involvement have informed research and provide models for the more common and pathophysiologically complex disorder CNO/CRMO.

Despite recent efforts, the exact molecular pathophysiology of CNO remains incompletely understood. Profound dysregulation of cytokine responses was demonstrated in $\mathrm{CNO} / \mathrm{CRMO}$. Failure to produce antiinflammatory cytokines interleukin (IL)-10 and IL-19 contributes to activation of inflammasomes and subsequent IL-1 $\beta$ release. In IL-10-deficient and in CNO-prone chronic multifocal osteomyelitis mice, IL-1 $\beta$ was linked to bone inflammation. Recently, increased inflammasome component expression and inflammasome assembly have been linked with CNO/CRMO in humans. Furthermore, alterations to the gut microbiome were suggested in contributing to IL-1 $\beta$ release from innate immune cells in mice, offering an interesting target in the search for molecular mechanisms in CNO.

This presentation will review molecular alterations in autoinflammatory bone disease, focussing on CNO and discuss therapeutic consequences. 
REFERENCES:

[1] Brandt D, Sohr E, et al. CD14+ monocytes contribute to inflammation in chronic nonbacterial osteomyelitis (CNO) through increased NLRP3 inflammasome expression. Clin Immunol. 2018 Nov;196:77-84.

[2] Hofmann SR, Schnabel A, et al. Chronic Nonbacterial Osteomyelitis: Pathophysiological Concepts and Current Treatment Strategies. J Rheumatol. 2016 Nov;43(11):1956-1964.

[3] Hofmann SR, Kubasch AS, et al. Altered expression of IL-10 family cytokines in monocytes from CRMO patients result in enhanced IL-1 $\beta$ expression and release. Clin Immunol. 2015 Dec;161(2):300-7.

[4] Zhao Y, Wu EY, et al. Consensus Treatment Plans for Chronic Nonbacterial Osteomyelitis Refractory to Nonsteroidal Antiinflammatory Drugs and/or With Active Spinal Lesions. Arthritis Care Res (Hoboken). 2018 Aug;70 (8):1228-1237.

[5] Ferguson PJ, Laxer RM. New discoveries in CRMO: IL-1 1 , the neutrophil, and the microbiome implicated in disease pathogenesis in Pstpip2-deficient mice. Semin Immunopathol. 2015 Jul;37(4):407-12.

[6] Cox AJ, Darbro BW, et al. Recessive coding and regulatory mutations in FBLIM1 underlie the pathogenesis of chronic recurrent multifocal osteomyelitis (CRMO). PLoS One. 2017 Mar 16;12(3):e0169687.

Disclosure of Interests: Christian Hedrich Grant/research support from: Novartis Pharmaceuticas for Research study on effector T cells in psoriasis, Speakers bureau: In 2016: Roche Pharmaceuticals, RheumatoLogisch, Dresden, Germany; Novartis, Advisory board $>$ Travel costs.

DOI: 10.1136/annrheumdis-2019-eular.8494

\section{\begin{tabular}{|l|l}
\hline SP0188 SAPHO - AN ADULT PERSPECTIVE & SA
\end{tabular}}

Gunter Assmann. University Medical School of Saarland, Medicine I, Oncology and Rheumatology, Homburg, Germany

Background: The acronym SAPHO has been introduced to describe a syndrome in adolescents and adults suffering from synovitis (arthritis), acne, pustulosis, hyperostosis, and non-bacterial osteitis preferentially in the sternal region. Currently, this also includes the entity $\mathrm{CNO}$ with non-bacterial chronic recurrent multifocal osteomyelitis (CRMO) in cases which primarily occur in adult age.

Objectives: The diagnostic certainty of SAPHO syndrome in case of incomplete presentation of the clinical features has been unclear so far. Furthermore, the treatment approach of SAPHO syndrome is difficult because the etiology remains unknown, although a reactive infectious osteitis in genetic predisposed subjects seems appealing.

Methods: Here we present relevant case series of SAPHO patients which should elucidate the relevance of different diagnostic procedures and treatment options. It has been noted, however, that particularly in respect of the use of antirheumatic drugs case series with predominantly small numbers of SAPHO patients suggest different treatment approaches.

Results: The diagnosis of SAPHO syndrome is made not only in the full picture of the disease according to the acronym, but also in non-bacterial and non-malignant osteitis with hyperostosis with simultaneous or delayed onset of acne, psoriasis and/or PPP. In addition, the primary manifestation of CNO in adulthood is nowadays classified as SAPHO syndrome. Recent studies have previously confirmed the usefulness of skeletal scintigraphy in 2 phases as a diagnostic. Imaging with MRI is frequently used preferentially for the assessment of osteitis activity, CT for the assessment of destruction and hyperostosis. The CT-guided biopsy of the lesion should be performed in solitary manifestation of osteitis. Basically, SAPHO syndrome has a high impact on patients' general health resulting in high burden of disease. There are many approaches to drug therapy, but only a few have been investigated in larger case series - none of them as controlled studies. Smaller case series of less than 10 patients reported limited efficacy of DMARD with MTX azathioprine, or ciclosporin. Studies with larger case numbers show a moderate efficacy of bisphophonates as well as TNF-alpha blockers. A short-term but not sustained effect has been demonstrated for the antibiotic azithromycin, as well as for steroids per os or as infiltration into osteitis. Referring to the treatment approach in SpA newly approved biologicals with IL-17 or IL-12/23 blocking effects demonstrated promising results in individual case reports of SAPHO patients.

Conclusion: Modern diagnostic imaging methods are increasingly being used for SAPHO syndrome. The selection of potentially effective drugs for SAPHO syndrome has increased. However, prospective studies to develop guidelines for the diagnosis and therapy of SAPHO syndrome are still lacking. Until further notice, the therapy is based on the recommendations for psoriatic arthritis or spondyloarthritis.

Disclosure of Interests: None declared

DOI: 10.1136/annrheumdis-2019-eular.8420

SATURDAY, 15 JUNE 2019

09:00:00 - 10:50:00

\section{EULAR Projects in clinical affairs}

\section{SP0189 \\ UPDATE ON EULAR RECOMMENDATIONS FOR THE MANAGEMENT OF SLE}

Antonis Fanouriakis. "Attikon" University Hospital, Rheumatology and Clinical Immunology, Athens, Greece

Background: Recent advances in treatment strategies and goals of treatment in systemic lupus erythematosus (SLE) called for an update of the EULAR recommendations for the disease, capitalizing on the strengths of and experience from similar previous projects.

Objectives: To update the EULAR recommendations for the management of SLE

Methods: Systematic literature review (01/2007-12/2017) followed by modified Delphi method to form questions, elicit expert opinions and reach consensus.

Results: Treatment in SLE aims at remission or low disease activity and prevention of flares. Hydroxychloroquine is recommended in all lupus patients, at a dose not exceeding $5 \mathrm{mg} / \mathrm{kg}$ real body weight. During chronic maintenance treatment, glucocorticoids should be minimized to less than $7.5 \mathrm{mg} /$ day (prednisone equivalent) and, when possible, withdrawn. Appropriate initiation of immunomodulatory agents (methotrexate, azathioprine, mycophenolate) can expedite the tapering/ discontinuation of glucocorticoids. In persistently active or flaring extrarenal dis ease, add-on belimumab should be considered; rituximab may be considered in organ-threatening, refractory disease. Updated specific recommendations are also provided for cutaneous, neuropsychiatric, haematological and renal disease. SLE patients should be assessed for their antiphospholipid antibody status, infectious and cardiovascular diseases risk profile, and preventative strategies be tailored accordingly.

Conclusion: The recommendations provide physicians and patients with updated consensus guidance on the management of SLE, combining evidencebase and expert-opinion.

Disclosure of Interests: Antonis Fanouriakis Paid instructor for: Amgen, GSK, Speakers bureau: Abbvie, Enorasis, Genesis Pharma DOI: 10.1136/annrheumdis-2019-eular.8473 